
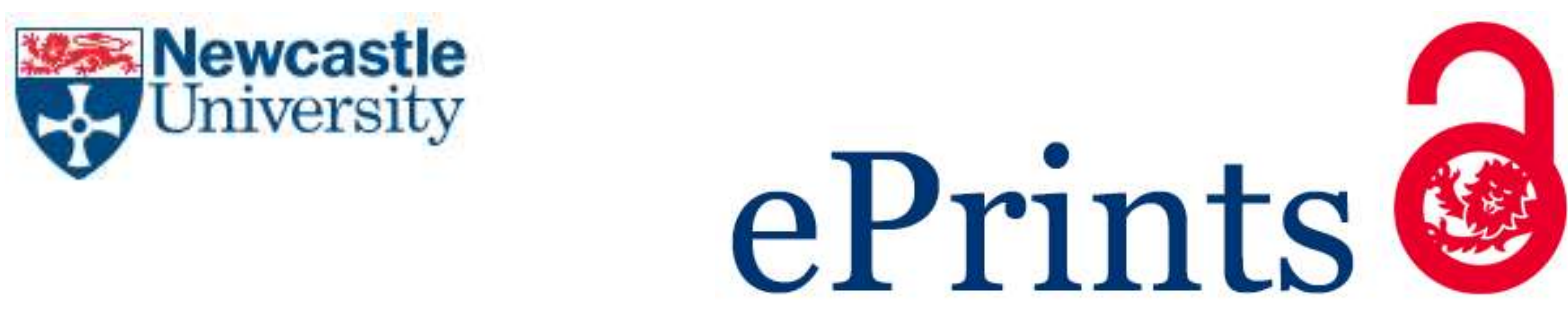

Rhyasen GW, Hattersley M, Yao Y, Dulak A, Wang W, Petteruti P, Dale I, Boiko S, Cheung T, Zhang J, Wen S, Castriotta L, Lawson D, Collins M, Bao L, Ahdesmaki MJ, Walker G, O'Connor G, Yeh T, Rabow AA, Dry J, Reimer C, Lyne P, Mills GB, Fawell S, Waring MJ, Zinda M, Clark E, Chen H. AZD5153: a novel bivalent BET bromodomain inhibitor highly active against hematologic malignancies. Molecular Cancer Therapeutics 2016, 15(8).

Copyright:

The final publication is available at AACR via http://dx.doi.org/10.1158/1535-7163.MCT-16-0141

Date deposited:

$30 / 08 / 2016$

Embargo release date:

16 August 2017

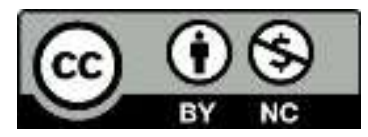

This work is licensed under a Creative Commons Attribution-NonCommercial 3.0 Unported License 


\section{AZD5153: a novel bivalent BET bromodomain inhibitor highly active against hematologic malignancies}

Garrett W. Rhyasen ${ }^{1 *}$, Maureen Hattersley ${ }^{1 *}$, Yi Yao ${ }^{1}$, Austin Dulak ${ }^{1}$, Wenxian Wang ${ }^{1}$, Philip Petteruti ${ }^{1}$, lan Dale ${ }^{2}$, Scott Boiko ${ }^{1}$, Tony Cheung ${ }^{1}$, Jingwen Zhang ${ }^{1}$, Shenghua Wen ${ }^{1}$, Lillian Castriotta $^{1}$, Deborah Lawson ${ }^{1}$, Michael Collins ${ }^{1}$, Larry Bao ${ }^{1}$, Miika J. Ahdesmaki ${ }^{2}$, Graeme Walker ${ }^{2}$, Greg O'Connor ${ }^{1}$, Tammie Yeh ${ }^{1}$, Alfred A. Rabow ${ }^{2}$, Jonathan Dry ${ }^{1}$, Corinne Reimer ${ }^{1}$, Paul Lyne ${ }^{1}$, Gordon B. Mills ${ }^{3}$, Stephen E. Fawell ${ }^{1}$, Michael J. Waring ${ }^{2}$, Michael Zinda ${ }^{1}$, Edwin Clark$^{1}$, Huawei Chen ${ }^{1}$

1. Oncology Innovative Medicines Unit, AstraZeneca R\&D Boston, 35 Gatehouse Drive, Waltham, MA, 02451, USA.

2. AstraZeneca, Mereside, Alderley Park, Macclesfield, Cheshire, SK10 4TG, U.K.

3. Department of Systems Biology, The University of Texas MD Anderson Cancer Center, Houston, TX 77030, USA.

${ }^{*}$ Co-first authors

\section{Conflict of Interest statement}

Garrett W. Rhyasen, Maureen Hattersley, Yi Yao, Austin Dulak, Wenxian Wang, Philip Petteruti, Ian Dale, Scott Boiko, Tony Cheung, Jingwen Zhang, Shenghua Wen, Lillian Castriotta, Deborah Lawson, Michael Collins, Larry Bao, Miika J. Ahdesmaki, Graeme Walker, Greg O'Connor, Tammie Yeh, Alfred A. Rabow, Jonathan Dry, Corinne Reimer, Paul Lyne, Stephen E. Fawell, Michael J. Waring, Michael Zinda, Edwin Clark, and Huawei Chen are employee of AstraZeneca and own AstraZeneca stocks. Gordon B Mills chairs the Scientific Advisory Board of AstraZeneca Oncology iMED, received honorarium as oncology speaker, and obtained sponsored research support from AstraZeneca.

\section{Running title}

Bivalent BRD4 inhibitor AZD5153 is active in hematologic cancers

\section{Correspondence}

Huawei Chen

Oncology Innovative Medicines Unit, AstraZeneca R\&D Boston, 35 Gatehouse Drive, Waltham, MA, 02451

+1781-839-4417

raymond.chen@astrazeneca.com 


\section{Abstract}

The bromodomain and extraterminal (BET) protein BRD4 regulates gene expression via recruitment of transcriptional regulatory complexes to acetylated chromatin. Pharmacological targeting of BRD4 bromodomains by small-molecule inhibitors has proven to be an effective means to disrupt aberrant transcriptional programs critical for tumor growth and/or survival. Herein, we report AZD5153, a potent, selective, and orally available BET/BRD4 bromodomain inhibitor possessing a bivalent binding mode. Unlike previously described monovalent inhibitors, AZD5153 ligates two bromodomains in BRD4 simultaneously. The enhanced avidity afforded through bivalent binding translates into increased cellular and antitumor activity in preclinical hematologic tumor models. In vivo administration of AZD5153 led to tumor stasis or regression in multiple xenograft models of Acute Myeloid Leukemia (AML), Multiple Myeloma (MM), and Diffuse Large B-cell Lymphoma (DLBCL). The relationship between AZD5153 exposure and efficacy suggests that prolonged BRD4 target coverage is a primary efficacy driver. AZD5153 treatment markedly impacts transcriptional programs of MYC, E2F, and mTOR. Of note, mTOR pathway modulation is associated with cell line sensitivity to AZD5153. Transcriptional modulation of MYC and HEXIM1 was confirmed in AZD5153treated human whole blood, thus supporting their use as clinical pharmacodynamic biomarkers. This report establishes AZD5153 as a highly potent, orally available BET/BRD4 inhibitor and provides rationale for clinical development in hematologic malignancies. 


\section{Introduction}

BRD4 is a member of the bromodomain and extraterminal (BET) family of chromatin reader proteins. BET proteins feature characteristic tandem $\mathrm{N}$-terminal bromodomains that function to interact with $\mathrm{N}$-acetyl lysine residues on histones and nuclear proteins (1-4). Bromodomainmediated interactions localize BRD4 to discrete chromosomal locations, facilitating the recruitment of transcriptional regulatory complexes, such as the general initiation cofactor Mediator and the Positive Transcription Elongation Factor b (P-TEFb) (5-7). Studies involving chromatin immunoprecipitation coupled with DNA-sequencing (ChIP-seq) have revealed a highly asymmetric genome-wide binding pattern of BRD4, with most chromatin-bound BRD4 localizing to enhancer elements. In particular, BRD4 has been shown to predominately bind enhancer elements, which are important for cell-type specification and oncogenesis $(8,9)$.

The discovery of lysine-mimetic small molecules capable of disrupting the chromatin binding activity of BET bromodomains was a crucial step towards uncovering the cancer supporting activity of BRD4 (10-12). In hematologic malignancies such as AML, MM and DLBCL, the misregulated expression of hematopoietic transcription factors is particularly important. BRD4 maintains and facilitates oncogenic transcription by interacting directly with transcription factors, and/or co-occupying transcription factor gene control loci, contributing to cancer cell proliferation and survival. For example, in AML, BRD4 chromatin occupancy is enriched at enhancer and promoter regions and highly correlated with the distribution of PU.1, FLI1, ERG, $\mathrm{C} / \mathrm{EBP} \alpha, \mathrm{C} / \mathrm{EBP} \beta$, and MYB (13). BRD4 inhibition interferes with the functional output of these lineage-specific transcription factors, and thereby inhibits $A M L$ maintenance. In MM the survival of malignant plasma cells is dependent on MYC transcriptional activity. MYC deregulation in MM can occur through gene translocation, amplification and overexpression $(14,15)$. In this setting BRD4 inhibition disrupts MYC activity and results in MM cell cycle arrest and senescence (16). DLBCL is a biologically and clinically heterogeneous disease, which is similarly typified by the activation of lineage-specific and growth-associated transcription factors including NF-kB, BCL6, p53, and MYC (17-20). BRD4 inhibition downregulates oncogenic MYC and E2F transcriptional pathways, resulting in antiproliferative effects across DLBCL subtypes (21). Based on this and other work, several drug candidates targeting BRD4/BET have progressed into clinical trials to treat Nut Midline Carcinoma (NMC), AML, Myelodysplastic Syndromes (MDS), MM, DLBCL, Glioblastoma Multiforme, and other solid tumors.

We recently described the discovery of BET bromodomain inhibitors that are chemically distinct from the commonly described benzodiazepine-based scaffold and capable of a novel bivalent binding mode (22). A dedicated medicinal chemistry campaign led to the discovery 
of a BET inhibitor clinical candidate AZD5153 (23). In this study, we characterize the in cell bivalent binding mode of AZD5153 and demonstrate the cellular and pharmacological advantages achieved through the unique biophysical properties of AZD5153 using AML, MM, and DLBCL as model systems.

\section{Materials and Methods \\ Cell lines and Reagents}

Most of cell lines were purchased from ATCC or DSMZ and a few obtained from academic laboratories from 2006 to 2014, and cultured according to providers' instructions (detailed information in Supplementary Table 1). Cell lines were authenticated using DNA fingerprinting short-tandem repeat (STR) assays and confirmed to be free of bacterial and viral contaminations by IDEXX (Westbrook, ME). All cell lines were used within15 passages, and less than 6 months. Antibodies used for western blot analysis were from Cell Signaling Technology (Danvers, MA): Anti-cMyc (\#5605), anti-GAPDH (\#2118), anti-phospho-p70S6K (T389/412) (\#9209), anti-p70S6K (\#2708), anti-PRAS40 (\#2610), anti-phospho-S6 (S235/236) (\#4856), anti-S6 (\#2217), anti-phospho-4EBP1 (S65) (\#9451), and anti-4EBP1 (\#9644).

\section{Bioluminescence Resonance Energy Transfer (BRET) NanoBRET assay}

The BRD4 NanoBRET assays have been previously described (24). Briefly, the expression construct of Histone H3.3-Halotag (NM_002107), was co-transfected with either NanoLucBRD4 full-length (1-1362) (060885), NanoLuc-BRD4 full-length N433F mutant, or NanoLuc$\mathrm{N}$-terminal BRD4-BD1 (amino acids 44-168) expression vector into HCT116 cells. Cells were then seeded into 384-well plates and treated with various concentrations of compounds. Plates were read after 18 hours of compound treatment.

\section{Immuno-fluorescent assays for BRD4 chromatin displacement}

The Immuno-fluorescent assays have been previously described (24). Briefly, BRD4 foci were stained by using anti-BRD4 antibody (Sigma Aldrich, HPA015 HPA015055) and secondary anti-rabbit antibody labeled with Alexa Fluor 488. The specificity of BRD4 antibody was verified by siRNA BRD4 knockdown in U2OS cells (24). Image acquisition was carried out by ImageXpress MicroXL High Content Screening System (Molecular Devices) and data analysis by MetaXpress (Molecular Devices).

\section{MYC Flow assay}


MM.1S cells were plated in 96-well plates at $200 \mathrm{k}$ cells/well and treated with serial concentrations of compound 1 hour after plating and grown for 16 hours at $37^{\circ} \mathrm{C}$. Cells were transferred into a 96 -well V-bottom plate and fixed sequentially with $2 \%$ paraformaldehyde for 10 minutes at $37^{\circ} \mathrm{C}$ followed with $90 \%$ cold methanol for 10 minutes on ice. Cells were washed and blocked in incubation buffer (PBS+0.5\%BSA) for 10 minutes and incubated with anti-cMyc primary antibody $(1: 200)$ at RT for 1 hour. Cells were washed twice and then stained with Alexa 488 conjugated anti-rabbit lgG (1:1000, Cell Signaling technology \#4412) for 30 minutes at RT and washed again. The pellet was resuspended in incubation buffer and analyzed by flow cytometer (BD FACSCalibur) using the FL-1 detector. Data was analyzed using FlowJo software V7. Live cell population was selected by gating on FCS/SSC and Geo Mean fluorescence of FL-1 signal was used to calculate $\mathrm{IC}_{50}$.

\section{Immunoblots}

Cells were plated at $1 \times 10^{6} \mathrm{cells} / \mathrm{ml}$ in 6 -well plates, treated with compounds at various concentrations for 4 or 24 hours. Crude lysate was prepared with $1 \times$ SDS lysis buffer $(60 \mathrm{mM}$ Tris- $\mathrm{HCl} \mathrm{pH} 6.8,1 \% \mathrm{SDS}$, and 10\% glycerol). For xenograft tumors, tissues were homogenized in buffer (20 mM Tris pH 8.0, $150 \mathrm{mM} \mathrm{NaCl}_{2}, 1 \% \mathrm{NP}-40$, in $10 \mathrm{ml}$ of lysis buffer supplement with $100 \mu \mathrm{l}$ of Phosphatase Inhibitor Cocktail2 (Sigma Cat \# P5726-5ML), $100 \mu \mathrm{l}$ of Phosphatase Inhibitor Cocktail3 (Sigma Cat \#P0044-5ML), 1 tablet of complete protease inhibitor cocktail (Roche \#11836153001). Samples with $20 \mu \mathrm{g}$ of total protein were loaded and western blots were run following typical procedures.

\section{Cell anti-proliferation and viability assays}

Sixty-one cell lines were cultured in appropriate medium. Optimal 384-well seeding densities were pre-measured for linear growth over 72 hours. $30 \mu \mathrm{l}$ cells/well were seeded in black, flat bottom 384-well plates and treated with compounds for 72 hours. The viable cells were measured using Alamar Blue reagents following manufacture's protocol (Invitrogen). The percent net growth is calculated by normalizing against the no-drug controls, and the $\mathrm{Gl}_{50}$ value, the concentration that causes $50 \%$ net growth inhibition, was calculated using GraphPad Prism 6.

Viability assay was performed following the same protocol as the anti-proliferation assay but differed in data treatment. Absolute number of remaining viable cells, instead of percent net growth, was determined after 72 hours of compound treatment. Cell viability $\mathrm{IC}_{50}$ value was obtaining by fitting the raw data points with sigmoidal nonlinear regression and $\mathrm{IC}_{50}$ curves were plotted using GraphPad Prism6.

\section{FACS apoptosis assay}


Apoptosis was analyzed by flow cytometry using CellEvent Caspase 3/7 Green detection reagent (ThermoFisher, \#C10423). MV-4-11, MM.1S and K562 cells were pre-treated with AZD5153 or I-BET762 for 48 hours in culture media. Cells were collected and stained with 5 $\mu \mathrm{M}$ final concentration of CellEvent for 30 minutes at $37^{\circ} \mathrm{C}$. Flow cytometry was done on a BD Fortessa using the Blue laser and FITC filter set. Gating and data analysis was done using FlowJo V7.0 software.

\section{RNA-sequencing}

After AZD5153 (200 nM) or vehicle treatment of hematological cell lines (AML - KG1A, MOLM13, MV-4-11, OCIAML2; MM - IM9, MM.1S, MOLP8, OPM2, RPMI8226; DLBCL OCILY19, WILL2) for 24 hours, RNA isolation, cDNA library generation, and sequencing to $12 \mathrm{M}$ reads on the Illumina HighSeq was carried out at $\mathrm{Q}^{2}$ Solutions (Morrisville, NC). Bcbionextgen was used to process FastQ files, perform quality control, alignment to genome build hg19, and quantify transcription expression based on Ensembl annotation. EdgeR [19910308] version 3.12.0 was used to identify differentially expressed genes using trimmed mean of $M$ values for normalization and the exactTest method for differential expression. All RNAseq data were deposited at GEO (GSE85840).

\section{Gene Set Enrichment Analysis}

Gene set enrichment analysis (GSEA) [16199517, 12808457] was performed using the GSEA software (Broad Institute, Cambridge, MA). A signal-to-noise preranked list of gene expression changes was generated from log2 FPKM values for vehicle and AZD5153-treated cell lines and compared to the hallmarks (h.all.v5.0.symbols.gmt) and transcription factor targets (c3.tft.v5.0.symbols.gmt) gene sets from MSigDb [16199517] using default parameters.

\section{Reverse phase protein microarray (RPPA) for proteomic profiling}

Proteomic profiling was carried out using high-density protein dot blot-based immunoassay system provided by Theranostics (Gaithersburg, MD). Cells were treated with $200 \mathrm{nM}$ AZD5153 for 24 hour, protein lysates prepared following Theranotics' protocol, and samples shipped to Theranostics to perform the array study. A total of 182 endpoints were assessed by immunostaining with corresponding antibodies. Data acquisition, quality control, and normalization were performed by Theranostics. AZD5153-modulated protein targets were identified by performing a paired, two-sided Student's T-test between DMSO and AZD5153 treated samples

\section{Quantitative PCR}


Cell pellets containing $\sim 1 \times 10^{6}$ cells or $\sim 10 \mathrm{mg}$ of tumor tissue were collected. RNA was extracted using an RNAeasy mini kit and manufacturer's protocol (Qiagen, cat\# 74104). RNA was converted to cDNA with a High Capacity RT kit following manufacturer's protocol (Applied Biosystems, cat\#4368814). PCR was performed using Taqman Gene Expression Master Mix (Applied Biosystems, cat\#4369016) with $300 \mathrm{ng}$ of cDNA and taqman probes (Applied Biosystems, \# MYC- Hs00153408_m1, \#HEXIM1-Hs00538918_s1, and \#GAPDH control4352934E) on ABI Prism 7900HT instrument.

\section{Efficacy and PK/PD studies in vivo xenograft models}

Female CB17 SCID and SCID beige mice were obtained from Charles River (Wilmington, MA) and NSG mice from Jackson Labs (Bar Harbor, ME). All animals were used in compliance with protocols approved by the Institutional Animal Care and Use Committees of AstraZeneca. Between $5 \times 10^{6}$ and $10^{7}$ tumor cells were implanted into appropriate host mice and tumor volume monitored twice weekly. Tumor volume $\left(\mathrm{mm}^{3}\right)$ is measured with calipers and calculated as $\left(W^{2} \times L\right) / 2$, where $W$ is width and $L$ is length. Tumor growth inhibition is calculated as $\left.\left(R T V_{\text {control }}-R T V_{\text {treatment }}\right)^{*} 100\right) /\left(R T V_{\text {control }}-1\right)$, where $R T V_{\text {control }}$ is the geometric mean of relative tumor volume of control group and RTV treatment the treatment group. Relative tumor volume is calculated using final tumor volume/initial tumor volume for individual animals. Statistical significance was evaluated using a one-tailed Student $t$ test. Eight to ten mice per group were used for efficacy and three mice per group for PD studies. Mice were treated with either vehicle $(0.5 \%$ hydroxymethylcellulose, $0.1 \%$ Tween 80$)$ or AZD5153 by oral gavage mini-pump infusion. For continuous administration of AZD5153, compound was solubilized in $20 \% \mathrm{v} / \mathrm{v}$ DMSO/60\% v/v HP-B-CD in water, loaded into a mini pump (Alzet, model 2002) and implanted subcutaneously in mice. Tumor fragments collected were snap frozen or fixed in $10 \%$ buffered formalin. Blood samples were collected from the same mice and stabilized in EDTA. Plasma concentrations were determined by liquid chromatography/tandem mass spectrometry method.

\section{Immunohistochemical (IHC) analysis}

Formalin fixed tumors were subjected to processing through graded ethanol, xylene and paraffin, embedded into paraffin blocks and $3 \mu \mathrm{m}$ sections were cut for assays. IHC was run with an optimized protocol on the Ventana Discovery XT using anti- Myc primary rabbit polyclonal antibody (Millipore \#06-340) at a 1:200 dilution, biotinylated anti-rabbit secondary antibody (Vector Labs \#PK-6101) and the DABMap detection kit (Ventana Medical \#760-124). For cleaved caspase 3, IHC was run with an optimized protocol on the Ventana Discovery XT using anti Cleaved Caspase 3 primary rabbit polyclonal antibody (CST-9661) at a 1:5000 dilution, biotinylated anti-rabbit secondary antibody (Vector Labs \#PK-6101) and the DABMap 
detection kit (Ventana Medical \#760-124). Digital slide images were acquired for both antibodies with the Aperio Scanscope XT using a 20X objective. Viable tumor areas were manually selected in Aperio Imagescope (version 11), and percent positive nuclei were quantified using a modified Nuclear Algorithm (version 9).

\section{Modulation of PD Biomarkers in human whole blood}

Whole blood was collected from six healthy donors and incubated with DMSO or $100 \mathrm{nM}$ AZD5153 for 2 hours at $37^{\circ} \mathrm{C}$. The whole blood was transferred to PAXgene RNA tubes (BD Diagnostics), incubated for 2 hours at room temperature for RNA stabilization and stored at $20^{\circ} \mathrm{C}$ until RNA extraction. RNA extraction was performed using PAXgene Blood RNA kits (Qiagen) according to manufacturer's instructions. Total RNA was then quantified, reverse transcribed and used as template for quantitative PCR using primers (ABI) specific for MYC and HEXIM1. Data was normalized using GAPDH as the housekeeping gene and converted to relative signal $\left(2^{-\Delta C t}\right)^{\star} 100$ to calculate percent of control.

\section{Results}

\section{AZD5153 is a potent BET bromodomain inhibitor possessing a bivalent binding mode} AZD5153, as shown in Figure 1A, is a potent and selective BET bromodomain inhibitor. AZD5153 induces dimerization of Bromodomain 1 construct (BD1) of BRD4 in X-ray co-crystal structure (23). Additional biophysical characterization by analytical ultracentrifugation and small angle X-ray scattering demonstrated that AZD5153 can bind bivalently, namely, that one molecule of AZD5153 binds and ligates two bromodomains in BRD4 (data not shown), in contrast to other reported BET inhibitors such as JQ1 and I-BET762 that bind monovalently with one molecule binding to each bromodomain in BRD4.

To confirm the in cell binding mode of AZD5153, we utilized a NanoBRET assay to monitor the interaction of BRD4 with chromatin using Halo-tagged histone $\mathrm{H} 3$ and a Nanoluciferase fusion protein with either the BD1 domain alone (BD1-BRD4) or a full-length BRD4 construct (FL-BRD4) (Figure 1B) (24). AZD5153 demonstrated a remarkable enhancement in potency for the displacement of full-length BRD4 relative to BD1, with $\mathrm{IC}_{50}$ values of $5.0 \mathrm{nM}$ and 1.6 $\mu \mathrm{M}$, for FL-BRD4 and BD1-BRD4, respectively (Figure 1C). In contrast, the monovalent inhibitor, I-BET762, displaced both BD1 and full-length protein with comparable potency (200 $\mathrm{nM}$ for BD1-BRD4 and $79 \mathrm{nM}$ for FL-BRD4) (22). Further evidence of simultaneous engagement of BD1 and BD2 by AZD5153 was obtained by analogous experiments comparing wild-type FL-BRD4 to a bromodomain N433F mutant in which the BD2 function was abolished. AZD5153 demonstrated a drastically reduced $\mathrm{IC}_{50}$ value of $1.4 \mu \mathrm{M}$ in displacing mutant BRD4 from chromatin, in contrast to $8.7 \mathrm{nM} \mathrm{IC}_{50}$ for wild-type protein (Supplementary 
Figure 1A). In comparison, the monovalent inhibitor JQ1 showed a similar chromatin displacement dose-response between the mutant and wild-type protein (Supplemental Figure 1B). Taken together, these data provide compelling evidence that the bivalent binding mode is the binding mechanism for AZD5153 in cells.

\section{Avidity of bivalent binding leads to enhanced cellular potency with increased BRD4 displacement from chromatin and MYC modulation}

We previously established a quantitative immuno-fluorescent assay to examine BRD4 chromatin displacement by measuring the disruption of BRD4 foci which are resulted from highly asymmetric binding of BRD4 to the enhancer regions (22). In this assay, AZD5153 potently disrupted BRD4 foci in U2OS cells with an $\mathrm{IC}_{50}$ value of $1.7 \mathrm{nM}$ (Figure 1D-E). In contrast, the monovalent inhibitor I-BET762 had an $\mathrm{IC}_{50}$ of $36.0 \mathrm{nM}$ in the same assay (Figure $1 \mathrm{E})$.

To assess whether enhanced BRD4 bromodomain binding activity translated into modulation of downstream targets, we examined MYC protein modulation in a mixed panel of 14 hematologic tumor cell lines. AZD5153 efficiently down-regulated MYC protein levels across the cell line panel irrespective of their sensitivity to AZD5153 (Supplementary Figure 2). AZD5153 was more potent than the monovalent inhibitor I-BET762 in down-regulating MYC protein, with $33 \mathrm{nM}$ of AZD5153 sufficient in reducing MYC to basal levels, whereas significant MYC remaining with $300 \mathrm{nM}$ of I-BET762 in MM1.S cells (Figure 1F). This observation was further confirmed by quantitative flow cytometry, which determined a MYC protein modulation $\mathrm{IC}_{50}$ of $5.4 \mathrm{nM}$ for AZD5153, compared to an $\mathrm{IC}_{50}$ of $329 \mathrm{nM}$ for I-BET762 (Figure 1G).

\section{Acute Myeloid Leukemia, Multiple Myeloma, and Diffuse Large B cell lymphoma cell lines are highly sensitive to AZD5153}

In order to assess the anti-proliferative activity of AZD5153 in hematologic cancers, we tested a cell-line panel comprised of $61 \mathrm{AML}, \mathrm{MM}$ and DLBCL cell lines. As shown in Figure 2A, all cell lines tested exhibited exquisite sensitivity towards AZD5153 $\left(\mathrm{GI}_{50}<150 \mathrm{nM}\right)$, with the majority of lines tested having a $\mathrm{GI}_{50}<25 \mathrm{nM}$. We hypothesized that the bivalent binding mode of AZD5153 could result in more robust target engagement and suppression of down-stream effector pathways, collectively leading to an enhanced reduction in cell viability in select hematological cancer subtypes. To test this hypothesis, five cell lines were treated AZD5153 or I-BET762 for 72 hours and cell viability was measured. Across all five cell lines, the AZD5153 IC 50 values were an average of 18-fold more potent than I-BET762 (Supplementary Figure 3), with representative cell viability $\mathrm{IC}_{50}$ curves shown in Figure 2B-D. 
Although treatments with both drugs essentially eliminated all viable cells in MM cell lines as evidenced by the higher drug concentration points in the dose-response curves (Figure 2C and Max\% cell kill in Supplementary Figure 4), AZD5153 reduced the level of remaining viable cells in KARPAS422, RSV411, and JURKAT lines when the maximal drug effect of AZD5153 and I-BET762 demonstrated a plateau (Figures 2B and 2D, and Max\% cell kill in Supplementary Figure 4). We deployed a flow cytometry assay to quantitatively measure caspase-3 positive cell populations in these cell lines to understand if a differential induction of apoptosis explained the difference observed in the cell viability assays. Unlike I-BET762, AZD5153 potently induces substantial apoptosis at $100 \mathrm{nM}$ in MV-4-11 and MM.1S cells (Figure 2E-F). In summary, the avidity of bivalent target engagement by AZD5153 translated into deeper cellular responses in hematological cell lines.

\section{AZD5153 modulates E2F, MYC, and mTOR transcriptional programs}

To gain further understanding of the underlying cellular mechanism of phenotypic response and to facilitate biomarker discovery, we performed RNA-sequencing on a panel of hematologic cancer cell lines ( $A M L n=4, M M n=5$, DLBCL $n=2)$ after treatment with AZD5153 (200 nM) or DMSO vehicle for 24 hours (GSE85840). RNA-sequencing analysis revealed 172 genes (109 down- and 63 up-regulated genes) that were significantly modulated (log2 fold change $>1$ and false discovery rate $(F D R)<0.05)$ across the cell line panel (Figure $3 \mathrm{~A}$ and Supplementary Table 1). Consistent with previous studies of BET inhibition, differential expression of HEXIM1, MYC, BCL2, FOSL1, and E2F1 was observed in select or multiple cell line types (16, 25-27). Gene Ontology (GO) analysis (28) was performed on the 109 uniformly down-regulated transcripts by AZD5153. Genes involved in G1/S transition and cell cycle progression were highly enriched, suggesting that the intersecting genes function in core cellular processes (Supplementary Figure 3). We used Gene Set Enrichment Analysis (GSEA) $(29,30)$ to gain a more comprehensive understanding of AZD5153 inhibitory effects on biological hallmarks and transcription factors using all cell line and tumor-specific data ranked by differential expression. Significant gene sets identified in at least one comparison (FDR < 0.1 ) were ranked and represented by a heat map. Similar correlating hallmarks were noted across all tumor types and included transcriptional targets of MYC, E2F transcription factors, and mTOR signaling components (Figures 3B - 3E).

\section{Repression of mTOR signaling is an AZD5153 sensitivity correlate}

In order to determine the consequences of BRD4 inhibition on protein levels, we used reversephase protein array (RPPA) technology to quantitatively examine the expression of 182 totaland phospho-proteins following 24 hours of AZD5153 treatment (200 nM) in K562, IM9, MOLP8, MV-4-11, and OCILY19 cell lines (Supplementary Table 2). To identify protein 
modulation that correlated with sensitivity to AZD5153, we segregated cell lines into resistant (K562, IM9) and sensitive (MOLP8, MV-4-11, OCILY19) groups based on a Gl 50 cutoff of 100 $\mathrm{nM}$. Interestingly, RPPA revealed that cell lines sensitive to AZD5153 exhibited a marked decrease in the level of mTOR-pathway associated proteins following AZD5153 treatment (Figure 4A). Selective down-regulation of p-p70S6K, PRAS40, and p-4EBP1 in AZD5153sensitive cell lines was confirmed by western blot with multiple doses of AZD5153 (Figure 4B). In contrast, MYC modulation was observed in both sensitive and resistant cell lines (Figure $4 B$ ), suggesting that MYC downregulation was not sufficient to modulate cell survival. These data suggest that in hematologic malignancies, mTOR pathway downregulation may serve as an appropriate biomarker of sensitivity to BRD4 inhibitors such as AZD5153.

\section{AZD5153 demonstrates potent anti-tumor activity in hematologic xenograft models}

In order to assess the in vivo activity of AZD5153, we explored the effects of dosing AZD5153 in AML, MM, and DLBCL xenografts. MLL-fusion leukemia has previously been shown to be dependent on BRD4 $(26,31)$. Thus we chose a xenograft model of MV-4-11, an AML cell line that harbors an MLL-AF4 fusion oncogene, for initial in vivo efficacy experiments. A dosedependent tumor growth inhibition (TGI) was observed for 1, 2.5, and $5 \mathrm{mg} / \mathrm{kg}$ daily (QD) oral doses of AZD5153, ranging from $72 \%$ TGI for $1 \mathrm{mg} / \mathrm{kg}$ to regression for $5 \mathrm{mg} / \mathrm{kg}$ (Figure $5 \mathrm{~A}$ and Supplementary Figure 5). A parallel experiment was carried out to further understand the dose-efficacy relationship for the lower end. Oral QD doses of $0.07,0.36$, and $0.72 \mathrm{mg} / \mathrm{kg}$ produced $16 \%, 23 \%$ and $53 \%$ TGI, respectively (Supplementary Figure 5). To determine if AZD5153 had anti-tumor activity beyond MLL-fusion AML, we tested it in additional xenograft models with known MYC or BRD4 linkage representing segments of MM, AML, and DLBCL. Treatment with $5 \mathrm{mg} / \mathrm{kg} \mathrm{QD}$, or a lower dose of $3.6 \mathrm{mg} / \mathrm{kg}$ for KARPAS422, resulted in $61 \%$ tumor regression in KMS11, 100\% TGI in MOLP-8, 98\% TGI in RS4;11, and 95\% TGI in KARPAS-422 xenograft models (Figures 5A-C and Supplementary Figure 5). Interestingly, the AML model KG1a demonstrates similar in vitro potency to AZD5153 as the above models; however, it is less sensitive to AZD5153 in vivo. For example, a higher dose of $10 \mathrm{mg} / \mathrm{kg}$ QD resulted in moderate TGI of 74\% (Figure 2A and Supplementary Figure 5). Understanding the difference between sensitivity in vitro and in vivo for KG1a would require further investigation. These data demonstrate AZD5153 is efficacious in a range of hematologic malignancies in vivo.

\section{Duration of BRD4 inhibition is the driver for efficacy and sustained BRD4 inhibition led to near complete tumor regression}

In an effort to understand if increased daily target coverage could drive greater efficacy, we evaluated QD, BID, and continuous dosing delivered via a subcutaneous mini-pump in MV-4- 
11 tumor-bearing mice for 14 or 24 days. Increased efficacy was observed when AZD5153 was given BID at 1.25 or $2.5 \mathrm{mg} / \mathrm{kg}$ (split dose) compared to 2.5 or $5 \mathrm{mg} / \mathrm{kg}$ QD (Supplementary Figure 5). The enhanced efficacy with BID dosing suggests that the duration of BRD4 target coverage is the driver for efficacy in the MV-4-11 tumor model. Consistent with this notion, near complete regression (97\%) of MV-4-11 tumors was achieved after 14 days of continuous delivery of AZD5153 at $12.8 \mathrm{mg} / \mathrm{kg} /$ week by mini-pump, with half of the dose at $6.4 \mathrm{mg} / \mathrm{kg} /$ week still resulting in tumor stasis (Figure 5D). A higher oral dose of 5 $\mathrm{mg} / \mathrm{kg} / \mathrm{daily}$, equivalent to $35 \mathrm{mg} / \mathrm{kg} / \mathrm{week}$, is needed to achieve a comparable efficacy to the $6.4 \mathrm{mg} / \mathrm{kg} /$ week continuous dose (Figure 5D). The steady and prolonged BRD4 inhibition achieved with the mini-pump was associated with increased apoptosis in the tumors compared with daily oral dosing after 7 days of treatment (Figure 5E-F). However, $12.8 \mathrm{mg} / \mathrm{kg} /$ week minipump group, that was twice the efficacious dose of $6.4 \mathrm{mg} / \mathrm{kg} / \mathrm{week}$, was accompanied by significant body weight loss with an average weight loss of $10 \%$ observed. Taken together, these results support that the duration of BRD4 inhibition is the primary driver for in vivo efficacy, and additional dose/schedule investigation is needed to determine an appropriate balance of target coverage and tolerability to maximize the therapeutic benefit.

\section{Pharmacodynamic evaluation in xenograft models and human whole blood supports MYC and HEXIM1 as biomarkers for AZD5153 inhibition}

To understand the pharmacokinetic (PK)/PD relationship of AZD5153, MYC mRNA and protein levels were measured by quantitative PCR (qPCR) and western blot. The concentration of AZD5153 was also measured in the plasma of the corresponding mouse after a single oral dose of $2.5,5$, or $10 \mathrm{mg} / \mathrm{kg}$ in MV-4-11 xenografts. All three doses induced significant modulation of MYC mRNA and protein over the first 8 hours but largely recovered by 16 hours (Figures $6 \mathrm{~A}$ and 6D). There was an inverse correlation between AZD5153 concentration in plasma and MYC mRNA levels in tumors (Figure 6A). Significant inhibition of MYC mRNA was achieved with free plasma concentration as low as $0.1 \mu \mathrm{M}$ of AZD5153 in mice with MV-4-11 tumors. The MYC mRNA levels returned to baseline as AZD5153 was cleared from the plasma. In contrast, HEXIM1 mRNA was up-regulated by AZD5153 treatment and had a similar but inverse PK/PD relationship (data not shown). An example of such a relationship is shown in Figure 6F, when mRNA levels of MYC and HEXIM1 were determined at 2 hours post the $5 \mathrm{mg} / \mathrm{kg}$ dose, MYC mRNA was down-regulated by about $80 \%$ whereas HEXIM1 mRNA was up-regulated by $400 \%$ (Figure $6 \mathrm{~F}$ ).

To confirm MYC protein modulation by AZD5153 and understand the heterogeneity within the tumors, immunohistochemistry (IHC) was performed in MV-4-11 tumors after $20 \mathrm{mg} / \mathrm{kg}$ dosing. As shown in Figure 6B and 6C, the vehicle treated tumor samples have $40 \%$ of cells 
with strong or medium MYC staining with the remaining $60 \%$ of cells having weak or no MYC staining. Acute oral administration of $20 \mathrm{mg} / \mathrm{kg}$ AZD5153 greatly reduced the number of cells with strong or medium intensity of MYC ( $97 \%$ and $75 \%$ reduction, respectively) at 2 hours post dosing. Correlating with PK, MYC staining intensity began to recover at 8 hours, the last time point taken in the study (Figures 6B and 6C). Interestingly, there were a significant number of cells with medium MYC staining remaining throughout the time points, highlighting the heterogeneity nature of response, a phenomenon that is frequently observed in the treatment of patient tumors.

Due to the limited availability of paired tumor biopsy samples during clinical development of novel agents, PD assessment with tumor tissue is not always achievable. Therefore we evaluated AZD5153 induced mRNA expression changes in whole blood as a surrogate PD endpoint. To identify potential clinical PD biomarkers, the mRNA expression of MYC and HEXIM1 genes were evaluated in human whole blood from healthy volunteers. Treatment with 100 nM AZD5153 for 2 hours resulted in 42\% decrease of MYC and 240\% increase of HEXIM1 mRNA (Figure 6E), similar to observation in MV-4-11 tumor tissue (Figure 6F). These data support the evaluation of these two biomarkers, together with other novel candidates that are under active investigation (manuscript in preparation), as potential human whole blood PD biomarkers in clinical trials.

\section{Discussion}

Herein, we assessed the characteristics of AZD5153, a novel BET bromodomain inhibitor. The bivalent binding mode of AZD5153 sets it apart from previously described BET bromodomain inhibitors. Mechanistically, the simultaneous ligation of both BRD4 bromodomains by AZD5153 allows for efficient displacement of BRD4 from chromatin at lower drug concentrations. This unique biophysical property translates into enhanced in vitro and in vivo pharmacological activity.

In the hematologic tumor setting, our comparative efficacy studies with QD, BID and continuous dosing of AZD5153 have revealed that duration of target coverage is the primary driver for in vivo efficacy. Twice daily dosing with the split QD dose mitigated the issue of fast clearance of AZD5153 from the mouse system and provided longer duration of target inhibition translating into better efficacy. We enhanced these findings by employing mini-pump drug infusion, which eliminates PK fluctuations, and provides consistent target inhibition. Compared to daily oral dosing, less than one fifth of AZD5153 was needed per week via mini-pump to achieve comparable efficacy. Furthermore, our data indicate $\mathrm{C}_{\max }$ does not play a significant 
role in driving AZD5153 efficacy. Namely, there was a greater than 12 fold difference in $\mathrm{C}_{\max }$ between $5 \mathrm{mg} / \mathrm{kg} /$ daily oral and $6.4 \mathrm{mg} / \mathrm{kg} /$ week mini-pump infusion. Unfortunately, enhanced efficacy achieved through prolonged target inhibition was often accompanied by significant body weight loss, which we used as a surrogate for gastrointestinal toxicity for BET inhibitors in mice. However, there remains a therapeutic index at lower doses using continuous infusion or a daily oral schedule, thus allowing for anti-tumor activity without concomitant bodyweight loss. Sustained BRD4 inhibition for a period of 2-3 days could lead to in vitro cell death of MV4-11 cancer cells via apoptosis, suggesting that an intermittent schedule is sufficient to deliver efficacy. Further investigation is needed to validate if such a schedule can sufficiently induce tumor cell death, while minimizing toxicity within normal tissues. Moreover, BET bromodomain inhibitors may have different scheduling requirements in solid tumor settings, owing to differences in tumor biology and microenvironment. A systematic investigation of both dose and schedule will be required to find the appropriate balance of drug exposure, efficacy, and tolerability for the myriad of disease subtypes for which BRD4 is being prosecuted as a cancer drug target. Ongoing clinical studies will no doubt be critical to define the optimal use of the BET bromodomain inhibitors.

Our gene expression analysis characterized the transcriptional response induced by AZD5153 in $\mathrm{AML}, \mathrm{MM}$, and DLBCL. Consistent with previously described studies examining BET bromodomain inhibitors, we observed significant modulation of MYC and E2F gene programs. However, our study is the first, to our knowledge, to reveal a significant modulation of an mTOR gene signature in these tumor types. Furthermore, we demonstrated that modulation of the mTOR signaling correlates with sensitivity to AZD5153 in cell lines we studied whereas MYC levels do not. Our data suggest that modulation of the mTOR pathway, which is widely de-regulated in many hematological cancers, contributes to the broad anti-cancer phenotype that we and others have observed for BET inhibitors in preclinical hematological cancer models. Consistent with this observation, the clinical candidate BET inhibitor, OTX015, exhibits synergy when combined with Everolimus in DLBCL (32). We speculate that downregulation of mTOR signaling components may serve as a correlative biomarker for future clinical studies of AZD5153 and/or other clinical candidate BET bromodomain inhibitors.

Both MYC mRNA and protein exhibited a concentration-dependent decrease in response to AZD5153 in both cell and in vivo models of hematological malignancies. Furthermore, inhibition of MYC mRNA was also observed in treated human whole blood and corresponded with an induction of HEXIM1 expression, supporting the use of these readouts as potential clinical biomarkers of target engagement for AZD5153. In the clinical setting, the use of human 
whole blood as a means to demonstrate target engagement could be beneficial, especially when paired tumor biopsies are not available.

In summary, AZD5153 is a novel BET bromodomain inhibitor with a bivalent binding mode. The unique biophysical properties of AZD5153 offer potency and pharmacological advantages over traditional monovalent BET bromodomain inhibitors. Based on potent in vivo anti-tumor activity, AZD5153 is a promising drug candidate for the treatment of hematological malignancies.

\section{Acknowledgments}

We thank Promega Corporation for performing mutant BRD4 cellular assays; Justin Cidado and Deepa Bhavsar for generating cell panel data; Galina Repik for assistance in cell line banking and retrieval.

\section{References}

1. Dhalluin C, Carlson JE, Zeng L, He C, Aggarwal AK, Zhou MM. Structure and ligand of a histone acetyltransferase bromodomain. Nature. 1999;399:491-6.

2. Moriniere J, Rousseaux S, Steuerwald U, Soler-Lopez M, Curtet S, Vitte AL, et al. Cooperative binding of two acetylation marks on a histone tail by a single bromodomain. Nature. 2009;461:664-8.

3. Dey A, Chitsaz F, Abbasi A, Misteli T, Ozato K. The double bromodomain protein Brd4 binds to acetylated chromatin during interphase and mitosis. Proceedings of the National Academy of Sciences of the United States of America. 2003;100:8758-63.

$4 . \quad$ Filippakopoulos P, Picaud S, Mangos M, Keates T, Lambert JP, BarsyteLovejoy D, et al. Histone recognition and large-scale structural analysis of the human bromodomain family. Cell. 2012;149:214-31.

5. Dey A, Nishiyama A, Karpova T, McNally J, Ozato K. Brd4 marks select genes on mitotic chromatin and directs postmitotic transcription. Molecular biology of the cell. 2009;20:4899-909.

6. Jang MK, Mochizuki K, Zhou M, Jeong HS, Brady JN, Ozato K. The bromodomain protein Brd4 is a positive regulatory component of $\mathrm{P}$-TEFb and stimulates RNA polymerase II-dependent transcription. Molecular cell. 2005;19:523-34.

7. Yang Z, Yik JH, Chen R, He N, Jang MK, Ozato K, et al. Recruitment of PTEFb for stimulation of transcriptional elongation by the bromodomain protein Brd4. Molecular cell. 2005;19:535-45.

8. Hnisz D, Abraham BJ, Lee TI, Lau A, Saint-Andre V, Sigova AA, et al. Superenhancers in the control of cell identity and disease. Cell. 2013;155:934-47.

9. Loven J, Hoke HA, Lin CY, Lau A, Orlando DA, Vakoc CR, et al. Selective inhibition of tumor oncogenes by disruption of super-enhancers. Cell. 2013;153:320-34. 10. Nicodeme E, Jeffrey KL, Schaefer U, Beinke S, Dewell S, Chung CW, et al. Suppression of inflammation by a synthetic histone mimic. Nature. 2010;468:1119-23. 11. Filippakopoulos P, Qi J, Picaud S, Shen Y, Smith WB, Fedorov O, et al. Selective inhibition of BET bromodomains. Nature. 2010;468:1067-73.

12. Shi J, Vakoc CR. The mechanisms behind the therapeutic activity of BET bromodomain inhibition. Molecular cell. 2014;54:728-36. 
13.

Roe JS, Mercan F, Rivera K, Pappin DJ, Vakoc CR. BET Bromodomain

Inhibition Suppresses the Function of Hematopoietic Transcription Factors in Acute Myeloid Leukemia. Molecular cell. 2015;58:1028-39.

14. Chng WJ, Huang GF, Chung TH, Ng SB, Gonzalez-Paz N, Troska-Price T, et al. Clinical and biological implications of MYC activation: a common difference between MGUS and newly diagnosed multiple myeloma. Leukemia. 2011;25:1026-35.

15. Brioli A, Melchor L, Walker BA, Davies FE, Morgan GJ. Biology and treatment of myeloma. Clinical lymphoma, myeloma \& leukemia. 2014;14 Suppl:S65-70.

16. Delmore JE, Issa GC, Lemieux ME, Rahl PB, Shi J, Jacobs HM, et al. BET bromodomain inhibition as a therapeutic strategy to target c-Myc. Cell. 2011;146:904-17. 17. Lenz G, Staudt LM. Aggressive lymphomas. The New England journal of medicine. 2010;362:1417-29.

18. Basso K, Dalla-Favera R. Roles of BCL6 in normal and transformed germinal center B cells. Immunological reviews. 2012;247:172-83.

19. Slack GW, Gascoyne RD. MYC and aggressive B-cell lymphomas. Advances in anatomic pathology. 2011;18:219-28.

20. Monti S, Chapuy B, Takeyama K, Rodig SJ, Hao Y, Yeda KT, et al. Integrative analysis reveals an outcome-associated and targetable pattern of p53 and cell cycle deregulation in diffuse large B cell lymphoma. Cancer cell. 2012;22:359-72.

21. Chapuy B, McKeown MR, Lin CY, Monti S, Roemer MG, Qi J, et al. Discovery and characterization of super-enhancer-associated dependencies in diffuse large B cell lymphoma. Cancer cell. 2013;24:777-90.

22. Waring MJ, Chen H, Rabow AA, Walker GE, Bobby R, Boiko S, et al. Bivalent binding to BET bromodomains. Nature Chemical Biology. 2016 - Accepted.

23. Bradbury RH CR, Carr GR, Chen H, Cornella-Taracido I, Feron L, Glossop S, et al. Optimization of a series of bivalent triazolopyridazine based BET bromodomain inhibitors: the discovery of (3R)-4-[2-[4-[1-(3-methoxy-[1,2,4]triazolo[4,3-b]pyridazin-6-yl)-4piperidyl]phenoxy]ethyl]-1,3-dimethyl-piperazin-2-one, AZD5153. Journal of Medicinal Chemistry. 2016 - In press, PMID: 27528113.

24. Machleidt T, WoodrPMID: 27528113oofe CC, Schwinn MK, Mendez J, Robers MB, Zimmerman K, et al. NanoBRET--A Novel BRET Platform for the Analysis of ProteinProtein Interactions. ACS chemical biology. 2015;10:1797-804.

25. Bartholomeeusen K, Xiang Y, Fujinaga K, Peterlin BM. Bromodomain and extra-terminal (BET) bromodomain inhibition activate transcription via transient release of positive transcription elongation factor $b(P-T E F b)$ from 7SK small nuclear ribonucleoprotein. The Journal of biological chemistry. 2012;287:36609-16.

26. Dawson MA, Prinjha RK, Dittmann A, Giotopoulos G, Bantscheff M, Chan WI, et al. Inhibition of BET recruitment to chromatin as an effective treatment for MLL-fusion leukaemia. Nature. 2011;478:529-33.

27. Baker EK, Taylor S, Gupte A, Sharp PP, Walia M, Walsh NC, et al. BET inhibitors induce apoptosis through a MYC independent mechanism and synergise with CDK inhibitors to kill osteosarcoma cells. Scientific reports. 2015;5:10120.

28. Chen EY, Tan CM, Kou Y, Duan Q, Wang Z, Meirelles GV, et al. Enrichr: interactive and collaborative HTML5 gene list enrichment analysis tool. BMC bioinformatics. 2013;14:128.

29. Subramanian A, Tamayo P, Mootha VK, Mukherjee S, Ebert BL, Gillette MA, et al. Gene set enrichment analysis: a knowledge-based approach for interpreting genomewide expression profiles. Proceedings of the National Academy of Sciences of the United States of America. 2005;102:15545-50.

30. Mootha VK, Lindgren CM, Eriksson KF, Subramanian A, Sihag S, Lehar J, et al. PGC-1alpha-responsive genes involved in oxidative phosphorylation are coordinately downregulated in human diabetes. Nature genetics. 2003;34:267-73.

31. Zuber J, Shi J, Wang E, Rappaport AR, Herrmann H, Sison EA, et al. RNAi screen identifies Brd4 as a therapeutic target in acute myeloid leukaemia. Nature.

2011;478:524-8. 
32.

Boi M, Gaudio E, Bonetti P, Kwee I, Bernasconi E, Tarantelli C, et al. The BET Bromodomain Inhibitor OTX015 Affects Pathogenetic Pathways in Preclinical B-cell Tumor Models and Synergizes with Targeted Drugs. Clinical cancer research : an official journal of the American Association for Cancer Research. 2015;21:1628-38.

\section{Figure Legends}

Figure 1. AZD5153 is a potent BET bromodomain inhibitor possessing a bivalent binding mode. (A) Chemical structure of AZD5153. (B) Schematic of cellular nano-BRET assay system using full-length BRD4 (FL-BRD4) or BD1 domain only BRD4 (BD1-BRD4) with a NanoLuc tag and histone H3.3 with a HaloTag in HCT116 cells. (C) Dose-response curves of AZD5153 displacing FL-BRD4 or BD1-BRD4 from histone H3.3 after 18 hours of treatment. (D) Formation of bright foci resulted from highly level of BRD4 bindings to the enhancer regions visualized by immuno-fluorescent staining in U2OS cells, and foci disruption by 4 hours treatment of $10 \mathrm{nM}$ AZD5153. (E) Dose-response curves of BRD4 foci inhibition with AZD5153 and a monovalent inhibitor I-BET762. (F) Western blots of MYC protein modulation by various concentrations of AZD5153 and I-BET762 in MM.1S cells with 4 hours of compound treatment. (G) MYC modulation dose-response curves of AZD5153 and I-BET762 in MM.1S cells by quantitative flow cytometry analysis.

Figure 2. AZD5153 exhibits broad anti-proliferative activity against hematological cancer cell lines. (A) Bar plot of $\mathrm{Gl}_{50}$ values across a mixed panel of $\mathrm{AML}$ (black), MM (orange) and DLBCL (red) hematological cancer cell lines following 72 hours treatment with AZD5153. Of note, *RS4;11 in AML panel is actually an acute lymphoblastic leukemia (ALL) line. B-D) Head-to-head comparison of cell viability dose-response curves of AZD5153 and I-BET762 in the indicated cell lines after 72 hours treatment. (E-F) Apoptosis induction was assessed by measuring caspase- 3 positive cell populations at various concentrations of AZD5153 and I-BET762 in the indicated cell lines 48 hours after compound treatment.

Figure 3. RNA-seq analysis reveals down-regulation of MYC, E2F, and mTOR target gene transcription by AZD5153. (A) Venn diagram representation of the most down- and up-regulated genes (FDR $<0.05$ and log2 fold change $>1$ ) following AZD5153 treatment for 24 hours in AML, MM, and DLBCL cell lines. (B) Heat maps of FDR $(q<0.01)$ values from GSEA of MSigDb transcription factor targets. Gene sets correlating with the AZD5153 phenotype are depicted. (C) Representative GSEA plots depicting gene sets most associated with AZD5153 down-regulation from MSigDb. (D) Heat maps of FDR $(q<0.01)$ values from GSEA of hallmarks gene set collections. (E) Representative GSEA plots depicting gene sets 
most associated with AZD5153 down-regulation from transcription factor target collections in all hematological cell lines.

Figure 4. Repression of mTOR signaling is an AZD5153 sensitivity correlate. (A) Unsupervised clustering heat map of mTOR-related total and phospho-proteins across five hematological cell lines from Reverse Phase Protein Array analysis. Bar at the top of the heat map indicates whether a cell line was sensitive (yellow) or resistant (blue) to AZD5153. Individual protein values were generated by calculating the ratio of protein modulation at 24 hour between DMSO and AZD5153 (200 nM) treatments. Turquoise, AZD5153-induced decreased expression; red, AZD5153-induced increased expression. Data are scaled according to $z$ scores by protein. (B) Western blot analysis of the change of protein level of select mTOR pathway signaling molecules and MYC by four concentrations of AZD5153 ( 0 , 2,20 , and $200 \mathrm{nM}$ ) after 24 hours of compound treatment across 5 hematological cell lines. Bar at the top of the Western blot indicates whether a cell line was sensitive (yellow) or resistant (blue) to AZD5153. Separation mark in the middle of Western blot indicates this is a combined blot from two gels. Only one representative GAPDH loading control is shown.

Figure 5. AZD5153 is active in hematological xenograft models. (A-C) Tumor growth curves with treatment of AZD5153 vs. vehicle in representative tumor xenograft models of AML, MM, and DLBCL. Mice were treated daily with vehicle or oral dose of 3.6 or $5 \mathrm{mg} / \mathrm{kg}$ AZD5153. (D) Comparative anti-tumor effects with oral dose of $5 \mathrm{mg} / \mathrm{kg} / \mathrm{daily}$, continuous delivery of $6.4 \mathrm{mg} / \mathrm{kg} / \mathrm{wk}$ or $12.8 \mathrm{mg} / \mathrm{kg} / \mathrm{wk}$ via mini-pump, and vehicle. Black lines, vehicletreated mice; blue, purple, and green lines, AZD5153-treated mice (E) Quantified cleaved caspase 3 intensity from immuno-histochemistry (IHC) in MV-4-11 tumors after 7 days of treatment. (F) Representative images of IHC used for image analysis showing increase in apoptoic cells after AZD5153 treatment

Figure 6. AZD5153 modulates MYC and HEXIM1 in AML xenograft tumors and human whole blood. (A) Relationship of MYC mRNA levels in MV-4-11 tumors and AZD5153 concentration in mouse plasma at three dose levels of AZD5153. Each bar represents an individual mouse. (B) Immunohistochemistry (IHC) quantifying percentage of MV-4-11 tumor cells with different levels of MYC staining intensity (weak, medium and strong) over time after a single oral $20 \mathrm{mg} / \mathrm{kg}$ dose of AZD5153. (C) Representative MYC IHC images used for analysis. The heterogeneity of MYC staining intensity is apparent in each tumor section. (D) Western blot for MYC expression in same tumors used for mRNA analysis shown in A. (E) Modulation of MYC and HEXIM1 mRNA in human whole blood after in vivo treatment with 100nM AZD5153. Blood samples were obtained from normal healthy volunteers. Error bars \pm 
SEM. (F) Modulation of MYC and HEXIM1 mRNA in MV-4-11 xenograft tumor tissue 2 hours post the $5 \mathrm{mg} / \mathrm{kg}$ oral dose. 